University of Louisville

ThinkIR: The University of Louisville's Institutional Repository

\title{
$5-2015$
}

\section{The impact of evaluative pressure and higher working memory capacity on sensorimotor skill performance.}

\author{
Lauren Grant \\ University of Louisville
}

Follow this and additional works at: https://ir.library.louisville.edu/honors

Part of the Cognitive Psychology Commons

\section{Recommended Citation}

Grant, Lauren, "The impact of evaluative pressure and higher working memory capacity on sensorimotor skill performance." (2015). College of Arts \& Sciences Senior Honors Theses. Paper 60.

http://doi.org/10.18297/honors/60

This Senior Honors Thesis is brought to you for free and open access by the College of Arts \& Sciences at ThinkIR: The University of Louisville's Institutional Repository. It has been accepted for inclusion in College of Arts \& Sciences Senior Honors Theses by an authorized administrator of ThinkIR: The University of Louisville's Institutional Repository. This title appears here courtesy of the author, who has retained all other copyrights. For more information, please contact thinkir@louisville.edu. 
The Impact of Evaluative Pressure and Higher Working Memory Capacity on Sensorimotor Skill Performance

By

\section{Lauren Grant}

Submitted in partial fulfillment of the requirements for Graduation summa cum laude and for Graduation with Honors from the Department of Psychological and Brain Sciences

University of Louisville

April, 2015 


\begin{abstract}
Underperformance in high-pressure situations, commonly known as choking under pressure, has been well-documented in the literature. For well-learned sensorimotor skills, such as sports, choking is thought to occur because individuals devote explicit attention to the steps of the skill, which disrupts performance. The current study examines how the type of pressure situation an individual experiences, and individual differences in working memory capacity, influence choking on a sensorimotor skill. Participants $(N=96)$ performed a Sensorimotor Reaction Time Task (SRTT) either under monitoring pressure, outcome pressure, or no pressure (control). High working-memory individuals performed significantly worse while completing the SRTT under monitoring pressure relative to outcome pressure. Low working-memory individuals performed marginally better than high working-memory individuals under monitoring pressure. These findings demonstrate that high working-memory negatively impacts sensorimotor skill performance in monitoring pressure situations. Furthermore, these results show how monitoring pressure leads to decreases in performance in high working-memory individuals due to enhancing the amount of attention these individuals direct to task execution.
\end{abstract}


The Impact of Evaluative Pressure and Higher Working Memory Capacity on Sensorimotor Skill Performance

Situations in which an individual experiences high pressure are inevitable throughout life. One oft-cited example is how standardized examinations are required in order for individuals to advance their career trajectories. The results of these exams often have significant consequences, whether it is being accepted to a university or obtaining a job certification. However, highpressure situations are not limited to traditional examinations. Performing well in a job interview or a high-stakes athletic performance can be just as critical for success. Interestingly, it is during instances such as these that individuals often perform beneath their usual capabilities. This phenomenon of underperforming during moments of high pressure is commonly referred to as choking under pressure (Beilock \& Gray, 2007). Out of the various explanations on how pressure impacts performance, the distraction and explicit monitoring theories are the most prominent within the current body of literature.

\section{Theories of Choking Under Pressure}

According to the distraction theory, individuals underperform in high-pressure situations because their attention is diverted from the task at hand by irrelevant thoughts. Rather than being able to apply the necessary amount of attention to successfully complete the task, distracting thoughts or worries cause individuals to perform worse than they normally would in a lowpressure situation (Beilock \& Carr, 2001, 2005; DeCaro, Rotar, Kendra, \& Beilock, 2010; Wine, 1971). Notably, the most support for this theory is found in the realm of academic performance (e.g., Beilock \& Carr, 2005). For example, Beilock, Kulp, Holt, and Carr (2004) had participants solve math problems of varying difficulty under both high and low pressure. As hypothesized, 
performance on the math problems that required the greatest amount of attention was the most negatively affected when participants were placed under high pressure.

In contrast, the explicit monitoring theory states that individuals in a high-pressure situation become increasingly self-aware of the steps they are taking to complete a skill. This increased attention hinders performance by interrupting the execution of well-learned, proceduralized processes that operate outside of attentional control (Beilock \& Carr, 2001). The majority of support for this theory comes from the realm of sensorimotor skills such as sports. For example, Gray (2004) demonstrated that highly skilled division I intercollegiate baseball players performed lower in a high-pressure condition than their baseline performance. However, they were able to judge the direction their bat was moving at specified intervals more accurately than during the baseline measurement. These results demonstrate that pressure causes individuals to be more aware of their movements, but in turn impairs their performance (Gray, 2004).

Both the distraction and explicit monitoring theories incorporate the idea that variations in the focus of attention can lead to choking under pressure (DeCaro, Thomas, Albert, \& Beilock, 2011). This concept of attention is a fundamental attribute of working memory. Working memory is simultaneously responsible for blocking out irrelevant details and maintaining a limited amount of information relevant to a task (Engle, 2002; Miyake \& Shah, 1999). According to the distraction theory, underperformance is caused by attention being diverted away from the task in a high-pressure situation such as taking an exam. In contrast, explicit monitoring theory suggests that when individuals are under pressure, they will increase their attention to skill execution to ensure optimum performance (Beilock \& Carr, 2001; DeCaro et al., 2011).

\section{Types of Pressure Situations}


Although explicit monitoring and distraction theories differ in their explanations for choking under pressure, they are both applicable depending on the type of situational pressure the individual is being placed under. DeCaro and colleagues (2011) identified two types of situational pressure an individual can be placed under: outcome pressure and monitoring pressure.

When individuals are placed under outcome pressure, they become very aware that a desirable result depends upon their performance of a particular task. Within psychological research, outcome pressure is often induced through the experimenter telling each participant that he or she has been paired with a partner and that, if each individual improves his or her score by a certain amount, each person in the pair will receive a monetary award. Each participant is then told that his or her partner already improved by the necessary amount. This therefore leaves it solely up to the participant to perform well enough to obtain the monetary award for both himself or herself and his or her partner (an important outcome). Outcome pressure is connected to the distraction theory in that the amount of working memory each individual is able to employ while solving problems is reduced, since the individual's attention is being diverted by worries of not performing well enough to receive the reward. Thus, outcome pressure is most likely to impact performance on tasks that require attention, such as academic skills (DeCaro et al., 2011).

In contrast, individuals under monitoring pressure become more self-conscious of their own actions while their performance is being evaluated. The use of an audience, video camera, or a mirror can all induce this particular kind of pressure situation (Carver \& Scheier, 1978; Davis \& Brock, 1975; Duval \& Wicklund, 1972; Geller \& Shaver, 1976). An example of how monitoring pressure is implemented in a laboratory setting is when an experimenter tells participants that they will videotape and watch their performance, so that others can evaluate 
how they perform the task. Due to the combination of the experimenter watching them and the video camera being nearby, the participants place more attention on the correct execution of the task than they normally would. Monitoring pressure relates to the explicit monitoring theory in that the individual becomes extremely self-conscious of his or her skill execution under highpressure. This self-consciousness is due to the skill execution being watched or evaluated. Thus, monitoring pressure is most likely to impact performance on well-learned sensorimotor skills that operate better without careful attention to the steps of performance, such as certain sports skills (DeCaro et al., 2011).

\section{Individual Differences in Working Memory Capacity}

Thus, research on these different kinds of pressure shows that pressure can have varying effects on the performance level of individuals depending on the attentional demands of the task being performed (DeCaro et al., 2011). It is also possible that individual differences in the ability, or tendency, to devote attention to a task may impact whether choking occurs.

Specifically, individuals differ in their working memory capacity—some individual devote more attention to a task, whereas other devote less attention (Engle, 2002).

Research has shown that individuals with higher working-memory capacity are impacted differently by outcome pressure on academic skills such as math (Beilock \& Carr, 2005; Beilock \& DeCaro, 2007). These studies found that individuals with high working-memory typically perform at a higher level, and are more likely to choke under pressure when distracted by thoughts or worries. Their results support the proposition that high working-memory individuals have more to lose under pressure. More specifically, their results posit that the magnitude of the drop in performance is larger for high working-memory individuals relative to low workingmemory individuals. 
However, research has not investigated how individual differences in working memory capacity impact the likelihood that individuals will choke in monitoring-pressure situations. Specifically, those with higher working memory might perform differently than those with lower working memory on sensorimotor skills, depending upon the kind of pressure situation they are placed in.

As noted above, although more attention-demanding performance strategies usually lead to higher accuracy, this is not always the case. Indeed, sometimes a simpler approach is the best route to performing a task. With increased expertise, certain tasks (such as sensorimotor skills) are better performed with less attention devoted to the individual steps of the skill (Beilock, 2007). In other words, as individuals increase their attention to executing the steps of the skill, they will actually perform worse. Interestingly, those with higher working memory often select complex task approaches when a simpler solution is more optimal for a task (DeCaro \& Beilock, 2010). It appears that individuals with higher working memory capacity often rely on their higher attention ability to perform complex tasks, even when the task does not call for a complex approach (DeCaro \& Beilock, 2010).

\section{Hypotheses}

With the knowledge that those with higher working memory tend to use more attentiondemanding strategies, and that monitoring pressure induces individuals to pay close attention to the steps of a skill, it can be hypothesized that those with higher working memory would do especially poorly at a well-learned sensorimotor task under monitoring pressure. As previously discussed, sensorimotor tasks are optimally performed when the step-by-step procedure becomes automatic. As such, placing individuals with higher working memory under monitoring pressure may lead them to overanalyze the completion of the sensorimotor task. In contrast, individuals 
with higher working memory may perform well under outcome pressure, because the distraction improves performance of a well-learned sensorimotor skill (cf. Beilock, Carr, MacMahon, \& Starkes, 2004).

Different effects are expected for individuals with lower working memory. Lower working-memory individuals can be predicted to perform better than higher working-memory individuals under monitoring pressure, because lower working-memory individuals have less capacity or a decreased tendency to pay close attention to a task. Performance for lower-working memory individuals should not differ between monitoring and outcome pressure conditions.

\section{Methods}

\section{Participants}

Participants $(N=96)$ were undergraduate students who completed the study in exchange for course credit. All participants performed a well-learned sensorimotor skill in one of three conditions: the low-pressure control condition $(n=37)$, an outcome pressure condition $(n=25)$, or a monitoring pressure condition $(n=34)$.

\section{Procedure}

Working Memory Capacity. Each participant first filled out a consent form, then was given the Automated Operation Span (AOspan) task in order to assess their working memory capacity. With the AOspan, participants are asked to solve a series of arithmetic equations (e.g., $(5 \times 2)-2=9$ ?) while simultaneously remembering a list of unrelated letters. The equationletter combinations are presented one at a time to the participant on the computer screen. Participants are then asked to verify whether the equation is correct and to remember the letter. At the end of a series of two to five of these sequences, participants are asked to recall the letters in the correct order (Unsworth, Heitz, Schrock, \& Engle, 2005). 
Sensorimotor Learning Task. Once the AOspan had been completed, participants completed the Serial Reaction Time Task (SRTT; Robertson, 2007). The SRTT is a sensorimotor learning task in which participants learn to press four keys on the computer keyboard in response to a shifting white square on the computer screen. Unknown to the participants, the probes often repeat a regular sequence. Over time, participants begin to learn this regular sequence by learning the associations between the key-presses (Robertson, 2007). Similar to other sensorimotor skills, this type of learning is thought to occur implicitly-outside of explicit attention (Robertson, 2007). Similarly, after these sequences are learned, they are typically thought to be executed automatically (Nissen \& Bullemeyer, 1987; Robertson, 2007). Each participant completed nine training blocks, with 12 repetitions of the sequence in each block. Each block also began and ended with 24 random trials.

Pressure Conditions. After training, participants completed a low-pressure test block under the same conditions as the training blocks. Performance on this block was used as a measure of obtained skill prior to the high-pressure block. This block was followed by a highpressure test block in one of three conditions. In the control condition, participants were simply asked to continue to try their best on the key-pressing task. In the outcome-pressure condition, the experimenter told each participant that he or she had been paired with a partner and that it was up to his or her performance to receive the monetary incentive for both of them (see description above). In the monitoring-pressure condition, the experimenter videotaped participants after telling them that the recordings of their performance would be watched by professors and students, as well as potentially being used in a film about research on basic skill learning for nationwide distribution (see DeCaro et al., 2011). 
SRTT Skill Scores. Performance on the SRTT was assessed by creating separate skill scores for the low-pressure and high-pressure tests. First, the median reaction time for each of the four sequences was averaged to create low-pressure and high-pressure sequence scores. Next, the median RT for each series of random trials was averaged to create low-pressure and highpressure random scores. The time to respond to random (i.e., non-sequence) trials is used to determine individual baseline speed. Finally, SRTT skill scores were calculated by subtracting the sequence score from the random score, and then dividing by the random score (i.e., [random - sequence] / random; Galea, Albert, Ditye, \& Miall, 2010). Higher skill scores indicate faster performance on the sequence relative to the random trials (i.e., learning of the sequence). Skill scores range from 0 (no learning of the sequence relative to random trials) to 1 (highest possible learning of the sequence, increased as the sequence approaches zero; DeCaro et al., 2011).

Questionnaires and Debriefing. After completing the final SRTT block, participants were asked to fill out a brief questionnaire concerning how much pressure they felt and their motivation to do well. The questionnaire was composed of two items, both of which incorporated a Likert scale. The first item asked participants to rank how important the task was to them, and the second item asked participants to rank how much pressure they felt while completing the task. After each of these items, participants were asked to explain why they chose that particular ranking.

Afterwards, participants were fully debriefed about the purpose of the experiment and were given an opportunity to ask questions. Participants in the outcome-pressure condition received the monetary incentive regardless of their performance level. 


\section{Results}

\section{Exclusion Criteria}

Participants were excluded from the dataset if they stated that they did not believe the high-pressure cover story $(n=1)$. Participants with greater than $20 \%$ errors on the math portion of the AOspan were also excluded $(n=5)$. Lastly, Participants with scores of $58(n=5)$ were excluded from the dataset. This value was chosen based on the norms provided by Unsworth et al. (2005).

Participants with a working memory score greater than 58 on the AOspan total score were categorized as high working-memory ( $n=62$ : control condition $n=25$, monitoring pressure $n=$ 21, outcome pressure $n=16$ ), and participants who had an AOspan total score below this value were categorized as low working-memory $(n=34$ : control condition $n=12$, monitoring pressure $n=13$, outcome pressure $n=9$ ).

\section{Pressure Magnitude}

A univariate analysis of variance (ANOVA) was performed to assess the amount of pressure the participants felt, based on condition (control, monitoring pressure, and outcome pressure), and working memory (high or low). A significant main effect of condition was found, $F(2,90)=4.02, p=.021$. No main effects of working memory $F(1,90)=1.19, p=.279$, or working memory $\mathrm{x}$ condition interaction, $F<1$, were found. As shown in Table 1 , a significant difference was found between the amount of pressure felt under outcome pressure and control conditions, $t(64)=-3.26, p=.002$, as well as between the outcome pressure and monitoring pressure conditions, $t(59)=2.34, p=.023$. No significant difference was found in the amount of pressure felt between the monitoring pressure and control conditions, $t(73)=-1.02, p=.311$. 
Thus, participants reported more pressure in the outcome pressure condition than the other two conditions, but this effect did not depend on working memory capacity.

\section{SRTT Performance}

SRTT performance in the high-pressure block was examined relative to the low-pressure block by computing a difference score (high-pressure skill score minus low-pressure skill score). Thus, higher scores on this variable indicate better performance under the high-pressure, relative to the low-pressure, block of this task. A 2 condition (control, outcome pressure, and monitoring pressure) x 2 working memory group (high, low) between-subjects ANOVA was conducted on this difference score. A significant main effect of condition was found, $F(2,90)=3.38, p=.038$. No main effect of working memory, $F<1$, or working memory x condition interaction, $F(2,90)$ $=1.14, p=.323$, were found.

Although the interaction was not significant, the small sample size was likely insufficient to detect effects, particularly for the low working-memory group. Thus, a series of simple effects tests were conducted to further test the hypotheses. First, an ANOVA was done to test the effect of condition for each working memory group. As shown in Figure 1, the effect of condition on the SRTT skill difference score was not significant for low working-memory participants, $F(2$, $31)=1.15, p=.329$. In contrast, the effect of condition was significant for high workingmemory individuals, $F(2,59)=4.31, p=.018$.

A series of planned comparisons revealed that high working-memory individuals performed significantly better under outcome pressure compared to the low-pressure control condition, $t(39)=-2.57, p=.014$. No significant difference was found for high working-memory participants between the control and monitoring pressure conditions, $t(44)=-.701, p=.487$. High working-memory participants performed significantly better under outcome pressure 
compared to monitoring pressure, $t(35)=2.35, p=.025$. The finding that the performance of high working-memory participants decreased under monitoring pressure relative to outcome pressure was consistent with my hypotheses.

A separate ANOVA was conducted to see if participants with high working-memory differed on the SRTT skill difference score from those with low working memory between conditions. There was a marginally-significant difference between individuals with low and high working-memory in the monitoring condition, $F(1,32)=3.10, p=.088$. Consistent with my hypotheses, low working-memory participants tended to outperform their high working-memory counterparts in the monitoring pressure condition. In contrast, no significant difference was found between individuals with low and high working memory in the control condition, $F<1$, or the outcome pressure condition, $F<1$.

\section{SRTT Skill Prior to High-Pressure Block}

The primary dependent measure used in this study was the difference in SRTT skill under the high-pressure block relative to the low-pressure block. This variable therefore accounts for individual differences in skill attained prior to the high-pressure block, and reveals differences in performance above and beyond prior skill level. However, it remains possible that high and low working-memory participants differ in their skill level prior to the high-pressure block. To examine this possibility, participants' low pressure skill scores were examined as a function of working memory group, using an ANOVA. A significant effect of working memory was found, $F(1,94)=4.71, p=.033$. High working-memory participants demonstrated significantly higher SRTT skill $(M=.059, S D=.109)$ than their low working-memory counterparts $(M=.008, S D=$ .110). A separate ANOVA revealed no differences in low pressure SRTT skill as a function of condition, $F<1$. 


\section{Discussion}

The present study examined how different factors that contribute to choking under pressure interact with individual differences in susceptibility to choking. Distraction theories state that pressure leads to distracted attention, whereas explicit monitoring theories suggest that pressure leads to enhanced attention to the steps of skill performance. Although distraction and explicit monitoring theories differ in their explanations as to why individuals choke under pressure, this study provides more support for the idea that each is applicable depending on the type of situational pressure an individual is placed under. Additionally, the findings of this study provide evidence that differences in working memory capacity play a fundamental role in how individuals perform under different forms of pressure.

A significant effect of condition on the SRTT skill difference score was found for high working-memory individuals. Most important, high working-memory participants were found to perform significantly worse under monitoring pressure compared to outcome pressure. High working-memory participants also performed somewhat (although not significantly) worse than low working-memory participants under monitoring pressure. The decreased performance of high working-memory participants under monitoring pressure relative to outcome pressure is consistent with my hypotheses. These findings suggest two possibilities. First, high workingmemory individuals may be overanalyzing the completion of the sensorimotor task, which is optimally performed when the step-by-step procedure becomes automatic. Second, high working-memory individuals may be benefitting by the distraction imposed in an outcomepressure condition, by preventing them from over-attending to the step-by-step procedures. 
Until this study, the relationship between individual differences in working memory and various types of pressure had not been investigated. In addition, the impact of working memory capacity on choking had not been studied for sensorimotor skills. For these reasons, these findings provide a significant contribution to the current literature, by highlighting how different types of pressure interact with working memory capacity to influence whether performance fails or succeeds.

Consistent with previous literature (Beilock \& Carr, 2005; Beilock \& DeCaro, 2007), high working-memory participants were shown to be more likely to choke. However, in these previous studies, choking was observed when high working-memory individuals completed a working memory-demanding math task. High working-memory individuals' superior working memory was disrupted by the distracting high-pressure condition (Beilock \& Carr, 2005; Beilock \& DeCaro, 2007). In the current study, the task performed (the SRTT) is thought to be performed better when executed outside of explicit attentional control, because the sequence becomes proceduralized with practice (much like a sports skill; Robertson, 2007). Thus, performance should be impaired when factors lead an individual to pay close attention to the steps of the skill. Thus, although consistent with previous work, the current findings demonstrate that high working-memory individuals can also choke by paying too much attention in a high-pressure situation.

Despite these interesting findings, there are three limitations of this study that must be addressed. First, the sample size for each condition is relatively low, particularly for low working-memory participants. I chose to divide participants into working memory groups based on published norms (Unsworth et al., 2005), rather than a median split, in order categorize individuals consistently with previous research. This categorization led to 62 high working- 
memory participants, but only 34 low working-memory participants across three pressure conditions. Thus, the findings for low working-memory participants cannot be closely interpreted, and the findings for high WM-participants must be treated with caution. Further research must be done to verify the results of this study.

Second, although participants reported feeling more pressure in the outcome pressure condition than control, pressure reports did not differ between monitoring pressure and control conditions. Thus, it is possible that differences between monitoring pressure and outcome pressure are due to differences in the magnitude of pressure felt more than the attention changes brought about by the high-pressure scenario. However, previous research (DeCaro et al., 2011) has shown no differences in pressure felt by individuals in these same pressure conditions, and has shown differences in performance on attention-demanding and proceduralized tasks in these conditions. In addition, no effects of working memory were found in this study, indicating that high and low working-memory groups reported similar feelings of pressure. A larger sample size would improve my ability to detect differences in pressure in the monitoring pressure condition, if they exist.

A third limitation is a potential confound with the SRTT skill level of the participants. The results of the analyses on the low-pressure skill score showed a significant main effect of working memory. Participants with high working-memory were more skilled than their low working-memory counterparts before the pressure manipulation took place. Therefore, it is unclear whether high working-memory participants performed significantly worse under monitoring pressure due to their greater ability to focus attention or due to their increased expertise. With increased expertise on procedural tasks, such as sports skills, individuals are more likely to choke under pressure by enhancing the attention they allocate to the steps of their 
skill (Beilock \& Carr, 2001; Gray, 2004). Future studies must be done in order to distinguish between these two possibilities.

In conclusion, this study provides an interesting demonstration of how individual differences in working memory are related to performance under varying forms of pressure. In particular, these results show that individuals with high working-memory tend to choke under monitoring pressure more so than low working-memory individuals. This evidence should facilitate future research on individual differences in regard to high-pressure situations, which would provide a clearer picture as to why performance under pressure differs between individuals. 


\section{References}

Beilock, S. L., \& Carr, T. H. (2005). On the fragility of skilled performance: What governs choking under pressure? Journal of Experimental Psychology: General, 130, 701-725. doi:10.1037/0096-3445.130.4.701

Beilock, S. L., \& Gray, R. (2007). Why do athletes “choke” under pressure? In G. Tenenbaum \& R. C. Ecklund (Eds.), Handbook of sport psychology (3rd ed., pp. 425-444). Hoboken, NH: Wiley.

Beilock, S. L., Kulp, C. A., Holt, L. E., \& Carr, T. H. (2004). More on the fragility of performance: choking under pressure in mathematical problem solving. Journal of Experimental Psychology. General, 133, 4, 584-600.

Bruya, B. (2010). Effortless attention: A new perspective in the cognitive science of attention and action. Cambridge, Mass: The MIT Press.

Carver, C. S., \& Scheier, M. F. (1978). Self-focusing effects of dispositional self-consciousness, mirror, presence, and audience presence. Journal of Personality and Social Psychology, 36, 324-332. doi:10.1037/0022-3514.36.3.324

Davis, D., \& Brock, T. C. (1975). Use of first person pronouns as a function of increased objective self-awareness and performance feedback. Journal of Experimental Social Psychology, 11, 381-388. doi:10.1016/0022-1031(75)90017-7

DeCaro, M. S., Thomas, R. D., Albert, N. B., \& Beilock, S. L. (2011). Choking under pressure: multiple routes to skill failure. Journal of Experimental Psychology. General, 140, 3, 390-406.

Engle, R. W. (2002). Working memory capacity as executive attention. Current Directions in Psychological Science, 11, 19-23. doi:10.1111/1467-8721.00160 
Galea, J. M., Albert, N. B., Ditye, T., \& Miall, R. C. (2010). Theta-burst TMS to the dorsolateral prefrontal cortex facilitates the consolidation of procedural skills. Journal of Cognitive Neuroscience, 22, 1158-1164. doi:10.1162/jocn.2009.21259

Geller, V., \& Shaver, P. (1976). Cognitive consequences of self-awareness. Journal of Experimental Social Psychology, 12, 99-108. doi:10.1016/0022-1031(76)90089-5

Gray, R. (2004). Attending to the execution of a complex sensorimotor skill: Expertise differences, choking, and slumps. Journal of Experimental Psychology: Applied. 10, 4254.

Lewis, B., \& Linder, D. (1997). Thinking about choking? Attentional processes and paradoxical performance. Personality and Social Psychology Bulletin, 23, 937-944. doi:10.1177/0146167297239003

Miyake, A., \& Shah, P. (1999). Models of working memory: Mechanisms of active maintenance and executive control. New York, NY: University Press.

Robertson, E. M. (2007). The serial reaction time task: Implicit motor skill learning? Journal of Neuroscience, 27, 10073-10075. doi:10.1523/JNEUROSCI.2747-07.2007

Unsworth, N., Heitz, R. P., Schrock, J. C., \& Engle, R. W. (2005). An automated version of the operation span task. Behavior Research Methods, 37, 3, 498-505.

Wine, J. (1971). Test anxiety and direction of attention. Psychological Bulletin, 76, 92-104. doi:10.1037/h0031332 


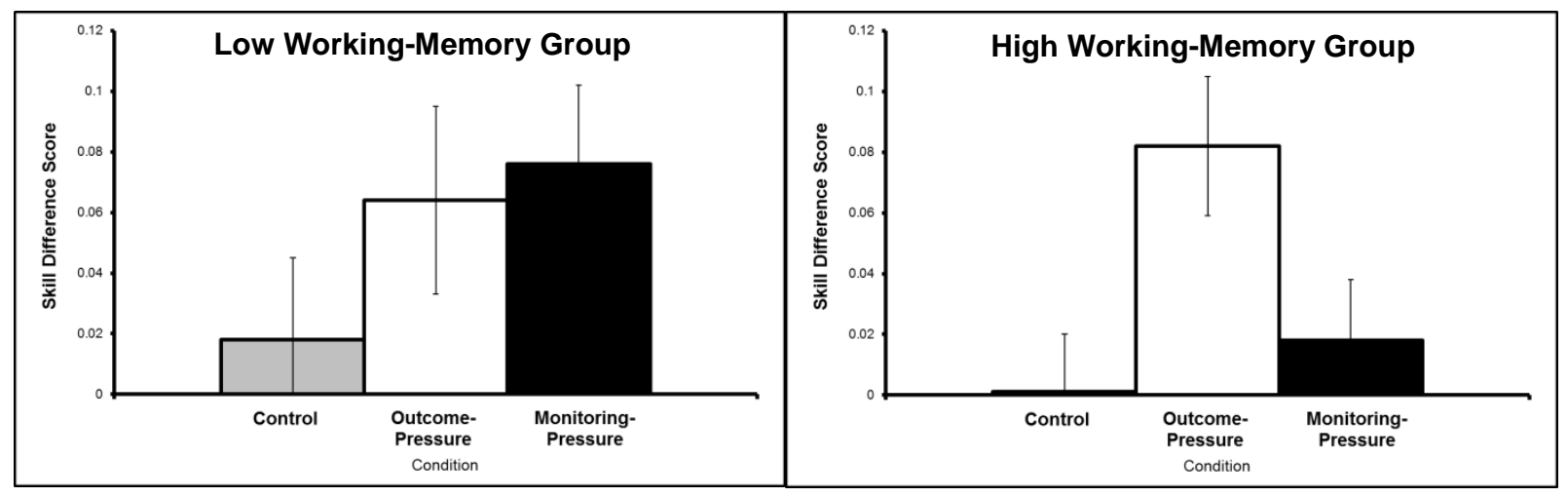

Figure 1. SRTT skill difference score (high-pressure test minus low-pressure test) as a function of condition and working memory group. Higher scores represent better performance under pressure (or control). Error bars represent standard errors. 


\begin{tabular}{|c|c|c|c|}
\hline WM Capacity & Control & Outcome Pressure & $\begin{array}{c}\text { Monitoring } \\
\text { Pressure }\end{array}$ \\
\hline High WM & 4.16 & 5.31 & 4.24 \\
\hline Low WM & 4.33 & 5.56 & 4.92 \\
\hline
\end{tabular}

Table 1. Magnitude of performance pressure felt (mean) for control, outcome pressure, and monitoring pressure conditions. 